Ethiopian Journal of Environmental Studies \& Management 10(4): 451 - 460, 2017.

ISSN:1998-0507

doi: https://dx.doi.org/10.4314/ejesm.v10i4.3

Submitted: November 11, 2016

Accepted: May 25, 2017

\title{
CONTRIBUTIONS OF VEHICLE INSPECTION OPERATIONS TO TRAFFIC SYSTEM IN ABUJA, NIGERIA
}

GBADAMOSI, K.T. AND ADENIGBO, A.J.*

Department of Transport Management Technology, Federal University of Technology, Akure, Nigeria

\begin{abstract}
The general view about the routine vehicle inspection operations is to ensure that vehicles are road worthy and meet safety requirements. This is done to enhance safe and clean transport within urban centres since the nature and condition of vehicles on roads can be associated with the efficiency of traffic system. This study examines the contributions of the routinevehicle inspection operations tothe overall traffic systemin Abuja, Nigeria. The study made use of secondary data collected from the records of the Directorates of Road Traffic Services (DRTS) on the number of vehicles inspected from 2007 - 2014 in Abuja. The study undertakes respondents' perception on the contributions of vehicle inspection operations to transport system using questionnaire designed to elicit information to achieve the objective of the study. A total of 142 respondents who were commercial taxi and bus drivers in Abuja were randomly sampled for the study. The study employed simple descriptive statistics and a combination of correlation and factor analysis as techniques for data analysis. The correlation analysis showed that the variables for considerations have positive relationships with one another except reduction in accident rates. The result of the factor loadings identified increased safety level (80.2\%) and reduction in the number of vehicles with poor parts (67.6\%) as the most significant contributions of the routine vehicle inspection operations to the overall transport system in Abuja. It concludes that vehicle inspection operations have other significant contributions to transportation system of urban centres aside ensuring road worthiness of vehicles.
\end{abstract}

Key Words: Vehicle Inspection, Transport System, Safety standards, Vehicle Road Worthiness

\section{Introduction}

Transportation system of urban centres is designed to provide effective movement of passengers and goods from one point to another. The design is such that it contributes substantially to the growth and development of nations because of its roles in the economic activities of cities. In Nigeria, the road transport sector remains the dominant mode in the movement of passengers, goods and services from one point to another within and across urban cities. Despite government's huge investments in the provision of road transport infrastructure in Nigeria, the road traffic 
system is characterised with poor performance in terms of vehicle administration, poor physical condition of vehicles, level of service, poor traffic and safety regulations (Filibus, 2012). This tells much on the level of efficiency of transport system in Nigeria.

Vehicle inspection is a process authorised by governments through which a vehicle is inspected to ensure that it conforms to regulations governing safety and emissions to ensure proper condition of vehicles on urban roads for effective movement of passengers and goods (Aruwa, 2014). In Nigeria, proof of inspection is required before a vehicle is registered or vehicle license can be renewed. According to Filibus (2012), vehicles are supposed to be driven and maintained by owners in accordance with road traffic regulations. The laws require certain standards of efficiency of most mechanical components of a vehicle to ascertain the level of its safety. Such components are the brakes, lights, horn, steering, windscreen, wipers, mirrors, exhaust pipes, tyres etc. These components must be properly inspected by inspection officers before vehicle registration. Alade (2012) identified the functions of vehicle inspection officers to include inspect and certify vehicle before registration or renewal of vehicle particulars, collaboration with companies with large fleets for on-site vehicle inspection to ensure compliance to safety regulations, and patrols on roads and highway to carry out routine checks and enforce compliance.

Many studies on vehicle inspection operations had been carried out in Nigeria in different dimension. Among such studies is the work of Aruwa (2014), Gana and Emmanuel (2014) which conclude that improper vehicle inspection and certification often results to road accidents. Oyeyemi (2003) also found that the operations of mechanically deficient vehicles may cause accident on roads. Adenigbo et al. (2016) found that vehicle inspection officers are faced with various operational challenges. Aruwa (2014) also found inadequate funding as the major source of challenges facing vehicleinspection operations in Abuja. Alade (2012) identified over focusing on the revenue generating aspect of vehicle inspection at the expense of safety in Nigeria as major challenge to vehicle inspections.

This study is an attempt to examine the contributions of vehicle inspection operations to the trafficsystem in Abuja. The efficiency in the overall transport system may reflect in clean and safe vehicular traffic on roads. Based on this, the study intends to find out if vehicle inspection operations contribute efficiently to transport system in Abuja. It is against this backdrop that this study examines possible contributions of vehicle inspection operations with a view to identifying the most significant contribution(s) to efficient traffic system in Abuja. The paper is structured such that section one deals with the introduction, section two is a description of the methodology while section three handles the results and discussion, and section four presents an highlight of the policy recommendation and conclusion.

\section{Methodology}

The study relied on both primary and secondary sources of data collection. Secondary data were extracted from the records of the Directorates of Road Traffic Services (DRTS), Abuja on the 
number and status of vehicles inspected in Abuja from 2007 - 2014. The primary data made use of survey of commercial taxi and bus drivers within the Federal capital Territory (FCT), Abuja through questionnaire administration. The questionnaires were administered at Kuje, Nyanya, Karu, Toyota, and Kubwa motor parks with the help of four trained research assistants. These are part of the major motor parks in FCT, Abuja. In all, a total of 142 respondents were sampled using the simple random technique. The sample size for the study is said to be adequate according to the suggestion of Hair et al. (1995) referred to in Williams et al. (2010) that sample sizes should be 100 or greater. The sampling technique ensures that the respondents were surveyed with equal chance of probability. This was meant to certify that the information obtained reflects the intents of the commercial drivers as regards the contributions of routine vehicle inspection operations to traffic system in Abuja. The instrument was designed on a multiple-item measurement fashioned on the 5-point Likert scale to allow for a wide measurement of the degree of the respondents' consideration of each contributions presented in the questionnaire. The variables were tabulated for the drivers to rank in order of significance from 1 - Not Significant to 5 - Highly Significant. The variables subjected to factor analysis are accident reduction, reduced number of vehicles with poor parts, reduced environmental pollution, on-road vehicle breakdown reduction, increased safety level, increased vehicle life span, proper vehicle maintenance culture, prevents abuse of vehicle capacity, detect fake vehicle documentation, improving fuel efficiency and increase resale value of vehicles.

Factor analysis was used as a technique for data analysis. This is in the light of the need to reduce the variables to a few orthogonal ones that could be used to explain the major contribution of vehicle inspection to efficient traffic system in Abuja. The main purpose of the factor analysis is to determine the number of common factors needed that can adequately describe the correlations between the observed variables, and estimating how each factor is related to each observed variable by estimating the factor loading (Oyesiku, 2000). A discussion of the model and application of factor analysis was presented by Laudau and Everitt (2004) as variable reduction technique. Ubogu (2013) and Adenigbo (2016) applied the model to study passenger choice of airport and cargo agents' choice of routing cargo respectively.

\section{Results and Discussion}

DRTS constantly conducts routine inspection operations where vehicles are randomly checked on roads. If faults are discovered, a defect notice will be issued in which case the owner will be required to have repairs made, and obtain a full inspection from a licensed examiner within 14 days to clear the notice. If serious safety violations are found, the vehicle's registration could be suspended on the spot and the operator will need to have the vehicle towed for litigation and payment of fines.

An increasing trend in the total number of vehicles inspected in Abuja is observed in Figure 1 from 2007 - 2014. This is in the sense that the total number of vehicles inspected stood at 65,397 in 
2007 and by 2014, it has increased to 145,537 vehicles. This might be as a result of increasing car ownership rate in Nigeria due to increasing disposable income and population as at 2014. Based on this, it is expected that the trend in the number of vehicles for inspection will continue to increase in the years ahead as income and urban migration rates increases. The status of vehicles inspected in Abuja from 2007 - 2014 shows the number of vehicles that failed and passed inspection test. Fig. 1 further shows an increasing trend in the number of vehicles that passed inspection tests and a decreasing trend for those that failed the tests.

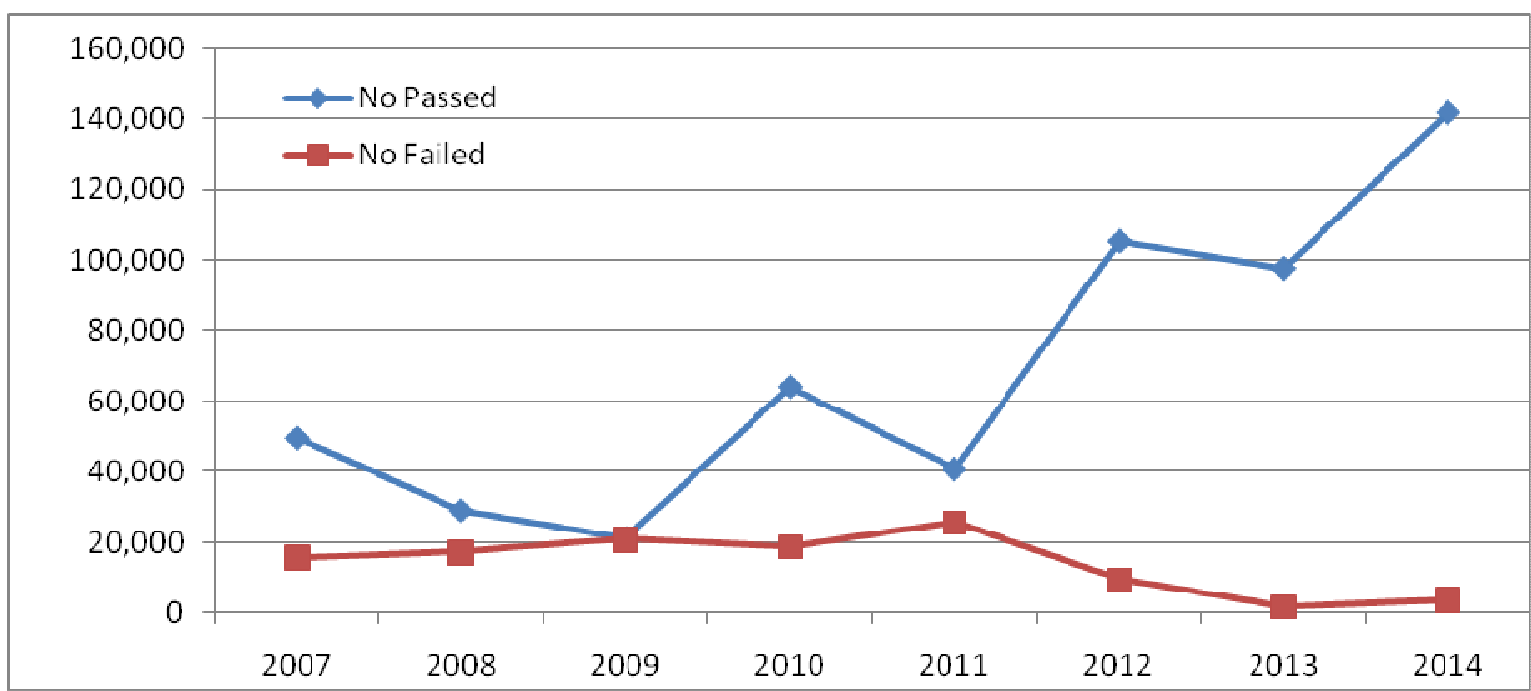

Fig 1: Number of vehicles Inspected in Abuja from 2007 - 2014

Source: DRTS, FCT

In order to assess the contributions of vehicle inspection operations to efficient traffic system in Abuja, some of the positive effects of vehicle inspection operations were examined in order to bring out the most significant ones. Factor analysis of the variables was carried out in order to determine the major ones among them.

It is normal that a data set to be used in statistics of this nature be subjected to test of suitability and adequacy. Therefore, to evaluate the suitability of the data, internal consistency checks were conducted using Kaiser-Meyer-Olkin (KMO) measure of sample adequacy and the Bartlett test of sphericity (Table 1). These tests were employed based on the requirement of factor analysis prior to factor extraction as suggested by Williams et al. (2010). The importance of the test is to determine if the samples were adequate to allow the use of factor analysis. 
Table 1: Result of KMO and Bartlett's Test

\begin{tabular}{lll}
\hline \multicolumn{2}{l}{ Kaiser-Meyer-Olkin Measure of Sampling Adequacy } & .937 \\
Bartlett's Test of & Approx. Chi-Square & 1687.023 \\
Sphericity & Df & 55 \\
& Sig. & .000 \\
\hline
\end{tabular}

The result in Table 1 shows a sampling adequacy value of $93.7 \%$ and significant at $\mathrm{p}<0.01$, which indicates that the data obtained is significantly adequate and suitable for the analysis. This is according to Cornish (2007) statement that a $\mathrm{KMO}$ result should be over $70 \%$ to be sufficiently correlated. Therefore, with a value above this threshold, the data are considered to be reliable for the use of factor analysis.

The possible contributions of vehicle inspection operations to efficient traffic system in Abuja are naturally dependent on one another by a degree of relationship that respondents attached to them. The correlation matrix (Table 2) of the variables under investigation reveals the nature and the extent of the relationship between the variables subjected to investigation in this study. For ease of table presentation, the variables are represented by AR, RVPP,
REP, RVB, ISL, VLSP, VMC, AVC, FVD, IFF, and RVV where $\mathrm{AR}=$ Accident Reduction; RVPP = Reduced Number of Vehicle with Poor Parts; REP $=$ Reduced Environmental Pollution; $\mathrm{RVB}=$ on Road Vehicle Breakdown; ISL $=$ Increased Safety Level; VLSP = Increased Vehicle Lifespan; VMC = Proper Vehicle Maintenance Culture; AVC $=$ Prevent Abuse of Vehicle Capacity; FVD = Detect Fake Vehicle Documentation; IFF = Improving Fuel Efficiency; and RVV = Increased Resale Value of Vehicles. It is observed that the correlation between all the variables shows a positive relationship except "AR - Accident Reduction" that has negative relationship with all other variables. This implies that any effect on one factor will produce a corresponding positive measure on the other variables except accident reduction with negative effect, which will produce a reverse effect.

Table 2: Correlation Matrix of the Effects of Vehicle Inspection on Traffic System

\begin{tabular}{llllllllllll}
\hline \multicolumn{1}{c}{ AR } & RVPP & REP & RVB & ISL & VLSP & VMC & AVC & FVD & IFF & RVV \\
\hline AR & 1.000 & & & & & & & & & & \\
RVPP & -.122 & 1.000 & & & & & & & & & \\
REP & -.141 & .724 & 1.000 & & & & & & & & \\
RVB & -.083 & .627 & .741 & 1.000 & & & & & & & \\
ISL & -.068 & .644 & .652 & .673 & 1.000 & & & & & & \\
VLSP & -.065 & .654 & .624 & .570 & .744 & 1.000 & & & & & \\
VMC & -.060 & .494 & .578 & .623 & .554 & .501 & 1.000 & & & & \\
AVC & -.033 & .602 & .671 & .619 & .634 & .604 & .559 & 1.000 & & & \\
FVD & -.042 & .603 & .575 & .574 & .680 & .558 & .483 & .645 & 1.000 & & \\
IFF & -.009 & .598 & .552 & .560 & .668 & .625 & .448 & .520 & .635 & 1.000 & \\
RVV & -.098 & .534 & .613 & .626 & .626 & .548 & .597 & .601 & .553 & .459 & 1.000 \\
\hline
\end{tabular}


The result as presented in Table 2 further indicates that 10 of the variables can be said to have strong and positive relationships with one another. The level of their relationship is said to be strong with $r$ having a threshold value greater than 0.500 . The weak correlated pair of variables exist between VMC and RVPP $(\mathrm{r}=0.494)$; FVD and VMC $(\mathrm{r}=0.483)$; IFF and VMC $(r=0.448)$; and RVV and IFF $(r=0.459)$. It should be noted that the said weak correlation has $r$ value greater than 0.400 , which indicates these variables are fairly related to influence one another.

Table 3 presents the communalities estimates of the variables after extraction shows that the item "Accident Reduction" (with 2.2\%) cannot be attributed to the three common factors that will form the major contributions of vehicle inspection operations to the overall transport system in Abuja. It implies that vehicle inspection operations in Abuja do not contribute to the reduction in road traffic accident as expected to enhance efficient traffic system within the city. This can be attributed to the fact that there exist other major factors that constitute road traffic accidents, and follows many findings that majority of road accidents are caused by human behaviour rather than mechanical faults.

Two (2) factors with eigen-values greater than one (1) were produced and accounted for $67.9 \%$ of the total variance of the variables (see Table 4). In determining the number of factors needed to represent the data set, the Kaiser's procedure of selecting the factor with eigen value greater than 1 criterion was adopted. The first factor accounted for $59 \%$ of the variance with eigen value of 7.138 while the other factor has $8.5 \%$ of the total cumulative variance with eigen value 1.019 .

Table 3: Communalities of the Effects of Vehicle Inspection on Traffic System

\begin{tabular}{lll}
\hline & Initial & Extraction \\
\hline Accident Reduction & .039 & .022 \\
Reduced number of vehicles with poor parts & .626 & .621 \\
Reduced environmental pollution & .699 & .728 \\
Road vehicle breakdown reduction & .656 & .698 \\
Increased safety level & .714 & .756 \\
Increased vehicle lifespan & .635 & .641 \\
Ensured proper vehicle maintenance culture & .485 & .524 \\
Prevent abuse of vehicle capacity & .599 & .612 \\
Detect fake vehicle documentation & .594 & .602 \\
Improving fuel efficiency & .556 & .648 \\
Increase Resale value of vehicles & .543 & .585 \\
\hline Extraction
\end{tabular}

Extraction Method: Principal Axis Factoring 
Table 4: Percentage of Total Variance Explained by

\begin{tabular}{llll}
\hline & \multicolumn{3}{l}{ Initial Eigen values } \\
\cline { 2 - 4 } Factor & Total & \% of Variance & Cumulative \% \\
\hline 1 & 7.138 & 59.485 & 59.485 \\
2 & 1.019 & 8.495 & 67.981 \\
3 & .729 & 6.078 & 74.059 \\
\hline
\end{tabular}

Extraction Method: Principal Axis Factoring

Table 5 shows the rotated factor matrix of the explanatory variables. In the table, three contributions were extracted to explain the underlying similarities of the 11 variables. The contributions were rotated using varimax rotation in order to maximise their orthogonality and clearly describe the pattern of the three major contributions of vehicle inspection operations on efficient traffic system in Abuja. Variables with absolute value of score below 0.4 were suppressed because in practice, according to Laudau and Everitt (2004), a largely arbitrary threshold value of 0.4 is often equated to 'high' loadings; in addition, variables were sorted by size (Table 5). It implies the greater the value the more significant is the variable as regards the contributions of vehicle inspection operations to traffic system in Abuja.

It can be seen from the Table 5 that all the 11 variables are loaded on the three extracted factors except "accident reduction". This implies that all the variables that loaded on the extracted factors are significant contributions of vehicle inspection operations to efficient traffic system in Abuja. It further shows that vehicle inspection operations do not contribute to reduction in road traffic accidents. This implies that road traffic accidents can occur if vehicles are road worthy and meet all safety regulations. Loaded on Factor 1 are all the variables except accident reduction. Factor 2 has reduced number of vehicles with poor parts, prevent abuse of vehicle capacity, reduce environmental pollution, reduce on-road vehicle breakdown rate, increase resale value of vehicles, and ensure proper vehicle maintenance culture; while Factor 3 loads on increased safety and detect fake vehicle documentation. All these variables are listed in order of significance as contributions of vehicle inspection operations to the overall transportation system in Abuja. 
Table 5: Rotated Factor Matrix of the effects of vehicle inspection in Abuja

\begin{tabular}{llll}
\hline & \multicolumn{3}{c}{ Factor } \\
Effects & 1 & 2 & 3 \\
\hline Increased safety level & .802 & & .416 \\
Improving fuel efficiency & .794 & & \\
Increased vehicle lifespan & .746 & & .445 \\
Detect fake vehicle documentation & .720 & & \\
Reduced number of vehicles with poor parts & .676 & .404 & \\
Prevent abuse of vehicle capacity & .616 & .482 & \\
Reduced environmental pollution & .590 & .616 & \\
On road vehicle break down reduction & .567 & .614 & \\
Increase resale value of vehicles & .505 & .574 & \\
Ensured proper vehicle maintenance culture & .450 & .567 & \\
Accident Reduction & - & - & \\
\hline
\end{tabular}

Extraction Method: Principal Axis Factoring.

Rotation Method: Varimax with Kaiser Normalization

Of the 11 variables identified and factor analysed, 10 loaded on Factor 1 and arranged by size according to their values. Increased safety level, improving fuel efficiency and increased vehicle life span loaded high with $80.2 \%, 79.4 \%$ and $74.6 \%$ respectively. Notwithstanding, it is pertinent to note that increased safety level with the highest value $(80.2 \%)$ is attributed to be the most significant contribution of vehicle inspection operations to the overall traffic system in Abuja. This can be as a result of the nature of the inspection operations requiring a thorough check on vehicles covering the engine, tyres, and other parts of the vehicles. These checks invariably tend to enhance safe movement of vehicles on roads. Inspection checks on safety level of vehicles have a relationship that tends towards reducing the number of vehicles with poor parts on roads, a variable that loads on both factor 1 and 2 with $67 \%$ and $40 \%$ respectively. As a result, it can be concluded that increased safety level and reducing number of vehicles with poor parts are the most significant contributions of vehicle inspection operations to efficient traffic system in Abuja. That the variable "Accident Reduction" does not load on any of the three extracted factors after suppressing all values less than 0.4 indicates that effective vehicle inspection operations does not have any contribution to reducing accidents on roads as expected. This result supports the study of Crain (1981) which noted that '.....vehicle inspection programs do not have the expected effect of reducing accident rates' (p 29) and that '.....more frequent inspections do not tend to reduce accident rates' (p 32).

\section{Policy Recommendation and Conclusion}

The contributions of vehicle inspection operations to efficient traffic system in cities can be said to be enormous. This calls for the need to put more efforts geared towards the development of vehicle inspection operations in Nigeria. The government should endeavour to promote the operations of vehicle inspection office (VIO) as it contributes to the efficiency 
of the overall transport system in Nigeria. This should be done such that campaigns on the need for vehicle owners and drivers to ensure all safety gadgets are well fitted in their vehicles as they are being used on roads. The government campaigns through its agencies such as VIO and Federal Road Safety Commission (FRSC) should include correct drivers attitude to reducing accidents as vehicle inspection operations do not contribute to accident reduction.

In conclusion, the study highlighted the number of vehicles inspected in Abuja from 2007 - 2014, and subjected 11 variables believed to be derived contributions vehicle inspection operations in Abuja to correlation and factor analyses. The study therefore concludes that vehicle inspection operations do not have significant effect as to the reduction in accident rates. It further highlighted increased safety level and reduced number of vehicles with poor parts on roads as the most significant contributions of vehicle inspection operations in Abuja.

\section{Acknowledgement}

The authors are grateful to the respondents that were surveyed for the purpose of this study. Many thanks also go to DRTS for providing the secondary data for the study.

\section{References}

Adenigbo, J.A. (2016). Factors Influencing Cargo Agents Choice of Operations in Abuja Airport, Nigeria. Journal of Air Transport Management 55: 113 - 119.

Adenigbo, A.J., Olorunfemi, S.O. and Aruwa, J. (2016). Challenges facing the Sustainability Operations of
Vehicle Inspection Officers in Abuja, Nigeria. In Ebohon, O.J., Ayeni, D.A., Egbu, C.O. and Omole, F.K. Procs. of the Joint International Conference (JIC) on $21^{\text {st }}$ Century Human Habitat: Issues, Sustainability and Development, 21-24 March 2016, Akure, Nigeria, page number 528533.

Alade, W.R. (2012). The Roles of Vehicle Inspection Officers $n$ Authentic Motor Vehicle Road Worthiness Certification. Paper Presented at The National Conference On Vehicle Road Worthiness Inspection And Testing Organized By National Automotive Council At Sheraton Hotel \& Towers, Abuja On 3rd \& 4th July, 2012.

Aruwa, J.T. (2014). Impact of Inspection Operations on Road Worthiness of Vehicles in Abuja. An Unpublished Master Thesis Submitted to Transport School, Nigerian Institute of Transport Technology (NITT), Zaria.

Crain, W.N. (1981). Vehicle safety inspections: How effective? American Enterprise Institute for Public Policy Research: Washington DC.

Cornish, K. (2007). Statistics: Factor Analysis. Mathematics Learning Support Centre. http://www.lboro.ac.uk/media/www lboroacuk/content/mlsc/downloads/ 3.3_Factoranalysis.pdf.

Filibus, B.E. (2012). The Roles of V.I.O's In Authentic Motor Vehicle Road Worthiness Certification Enforcement. A Paper Presented At The National Conference On 
Vehicle Road Worthiness Inspection and Testing Organized By National Automotive Council At Nicon Luxury Hotel, Abuja.

Gana, A.J., Emmanuel, J.A. (2014). Road Transportation and Traffic Law Enforcement in Nigeria: A case study of the Federal Road Safety Corps (FRSC).West African Journal of Industrial \& Academic Research, 11(1): 134-151.

Hair, J., Anderson, R.E., Tatham, R.L. and Black, W.C. (1995). Multivariate Data Analysis. 4th ed. New Jersey: Prentice-Hall Inc.

http://mlsc.lboro.ac.uk/resources/statistic s/Factoranalysis.pdf.

Laudau, S. and Everitt, B. (2004). A Handbook of Statistical Analyses using SPSS. Chapman and Hall/CRC Press, New York.
Oyesiku, K.O. (2000). Fundamentals of Research Methodology. HEBP, Lagos.

Ubogu, A.E. (2013). Determinants of Passengers' Choice: A Case Study of Mallam Aminu Kano International Airport (Nigeria). International Journal of Traffic and Transport Engineering 3(3): 230 242.

DOI: http://dx.doi.org/10.7708/ijtte.2013. 3(3). 01

Williams, B., Brown, T. and Onsman, A. (2010). Exploratory Factor Analysis: A Five-Step Guide for Novices. Australasian Journal of Paramedicine, 8(3). Retrieved from http://ro.ecu.edu.au/jephc/vol8/iss3/ 1 